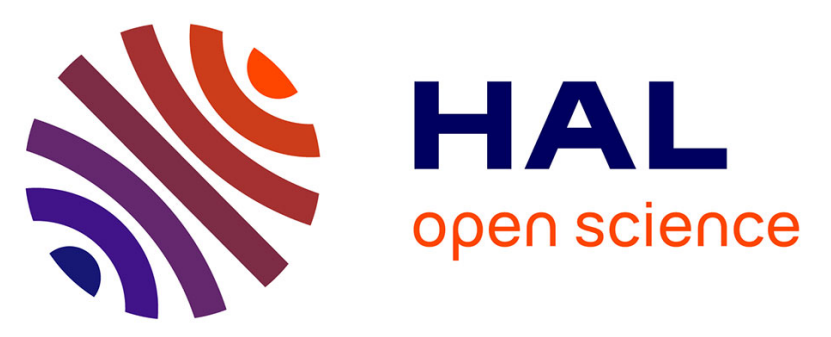

\title{
Associations between untargeted plasma metabolomic signatures and gut microbiota composition in the Milieu Intérieur population of healthy adults
}

Valentin Partula, Mélanie Deschasaux-Tanguy, Stanislas Mondot, Agnès

Victor-Bala, Nadia Bouchemal, Lucie Lecuyer, Christine Bobin-Dubigeon,

Marion Torres, Emmanuelle Kesse-Guyot, Bruno Charbit, et al.

\section{To cite this version:}

Valentin Partula, Mélanie Deschasaux-Tanguy, Stanislas Mondot, Agnès Victor-Bala, Nadia Bouchemal, et al.. Associations between untargeted plasma metabolomic signatures and gut microbiota composition in the Milieu Intérieur population of healthy adults. British Journal of Nutrition, 2020, 126 (7), pp. 982-992. 10.1017/S0007114520004870 . hal-03073792

\section{HAL Id: hal-03073792 \\ https://hal.science/hal-03073792}

Submitted on 29 Dec 2020

HAL is a multi-disciplinary open access archive for the deposit and dissemination of scientific research documents, whether they are published or not. The documents may come from teaching and research institutions in France or abroad, or from public or private research centers.
L'archive ouverte pluridisciplinaire HAL, est destinée au dépôt et à la diffusion de documents scientifiques de niveau recherche, publiés ou non, émanant des établissements d'enseignement et de recherche français ou étrangers, des laboratoires publics ou privés.

\section{(ㄷ)(1) $\$$}

Distributed under a Creative Commons Attribution - NonCommerciall 4.0 International 
Associations between untargeted plasma metabolomic signatures and gut microbiota composition in the Milieu Intérieur population of healthy adults

Valentin PARTULA ${ }^{1,2} *$, Mélanie DESCHASAUX-TANGUY ${ }^{1 *}$, Stanislas MONDOT ${ }^{3}$, Agnès VICTOR-BALA ${ }^{4}$, Nadia BOUCHEMAL ${ }^{4}$, Lucie LECUYER ${ }^{1}$, Christine BOBIN-DUBIGEON ${ }^{5,6}$, Marion J. TORRES ${ }^{7}$, Emmanuelle KESSE-GUYOT ${ }^{1}$, Bruno CHARBIT ${ }^{8}$, Etienne PATIN ${ }^{9}$, Karen E. ASSMANN ${ }^{1}$, Paule LATINO-MARTEL ${ }^{1}$, Chantal JULIA ${ }^{1,10}$, Pilar GALAN ${ }^{1}$, Serge HERCBERG $^{1,10}$, Lluis QUINTANA-MURCI ${ }^{9}$, Matthew L. ALBERT ${ }^{11}$, Darragh DUFFY ${ }^{12}$, Olivier LANTZ $^{13,14}$, Philippe SAVARIN ${ }^{4}$, Mohamed Nawfal TRIBA ${ }^{4}$, Mathilde TOUVIER ${ }^{1}$, The Milieu Intérieur Consortium ${ }^{15}$

* co-first authors

\section{Affiliations:}

${ }^{1}$ Sorbonne Paris Nord University, INSERM, INRAE, CNAM, Nutritional Epidemiology Research Team (EREN), Epidemiology and Statistics Research Center - University of Paris (CRESS), Bobigny, France

${ }^{2}$ University of Paris-VII Denis Diderot, Université de Paris, Paris, France

${ }^{3}$ MICALIS Institute (Inrae/AgroParisTech), Jouy-en-Josas, France

4 Chemistry, Structure, and Properties of Biomaterials and Therapeutic Agents CSPBAT, Nanomedicine, Biomarkers and Detection Team (CNRS U7244/Université Sorbonne Paris Nord), Bobigny, France

${ }^{5}$ Department of Biopathology, Institut de Cancérologie de l’Ouest, Saint-Herblain, France ${ }^{6}$ Sea, Molecules, Health MMS EA2160 (CNRS FR3473/Université de Nantes), Nantes, France

7 Nutritional Surveillance and Epidemiology Team (ESEN), French Public Health Agency, Sorbonne Paris Nord University, Epidemiology and Statistics Research Center - University of Paris (CRESS), Bobigny, France

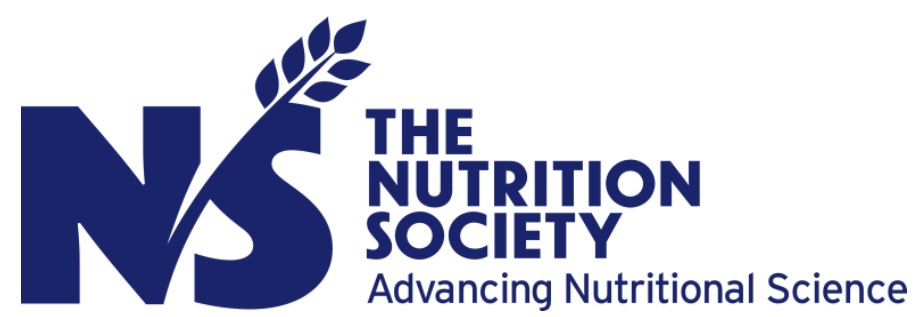

This peer-reviewed article has been accepted for publication but not yet copyedited or typeset, and so may be subject to change during the production process. The article is considered published and may be cited using its DOI

10.1017/S0007114520004870

The British Journal of Nutrition is published by Cambridge University Press on behalf of The Nutrition Society 
Accepted manuscript

${ }^{8}$ Centre for Translational Research, Institut Pasteur, Paris, France

${ }^{9}$ Human Evolutionary Genetics laboratory (CNRS URA3012/Institut Pasteur), Paris, France

${ }^{10}$ Department of Public Health, AP-HP Paris Seine-Saint-Denis University Hospital System, Bobigny, France

${ }^{11}$ Department of Immunology and Infectious Diseases, Insitro, San Fransisco, CA 94080, USA

${ }^{12}$ Immunobiology of Dendritic Cells laboratory (Inserm U1223/Institut Pasteur), Paris, France

${ }^{13}$ Curie Institute, PSL University, Inserm U932, Paris, France

${ }^{14}$ Clinical Investigation Center CIC-BT1428 (Institut Gustave Roussy/Institut Curie), Inserm, Paris, France

${ }^{15}$ The Milieu Intérieur Consortium, Pasteur Institute, Paris, France. Additional information can be found at: http://www.milieuinterieur.fr/en

Corresponding author: Mélanie DESCHASAUX-TANGUY

m.deschasaux@eren.smbh.univ-paris13.fr

EREN SMBH Université Sorbonne Paris Nord

74 rue Marcel Cachin, F-93017 Bobigny Cedex

Running head: Metabolomic signatures and gut microbiota

Keywords: Gut microbiota; host-gut microbiota relationships; NMR metabolomics; cross-sectional population-based study; epidemiology.

Study registration: NCT01699893

Abbreviations: Carr-Purcell-Meiboom-Gill, CPMG; False discovery rate, FDR; Mass spectrometry, MS; Milieu Intérieur, MI; Multivariate analyses with linear models, MaAsLin; Nuclear magnetic resonance, NMR; 1H 1D NMR pulse sequence Nuclear Overhauser Effect Spectroscopy, NOESY1D; Operational taxonomic units, OTU; Permutational analysis of variance, PERMANOVA; Polymerase chain reaction, PCR; Principal Component Partial R-square, PC-PR2; Standard deviation, SD; Total correlated spectroscopy, TOCSY; Trimethylamine, TMA; Trimethylamine N-oxide, TMAO. 


\section{ABSTRACT}

Host-microbial co-metabolism products are being increasingly recognized to play important roles in physiological processes. However, studies undertaking a comprehensive approach to consider hostmicrobial metabolic relationships remain scarce. Metabolomic analysis yielding detailed information regarding metabolites found in a given biological compartment holds promise for such an approach. This work aimed to explore the associations between host plasma metabolomic signatures and gut microbiota composition in healthy adults of the Milieu Intérieur study. For 846 subjects, gut microbiota composition was profiled through sequencing of the 16S rRNA gene in stools. Metabolomic signatures were generated through proton nuclear magnetic resonance analysis of plasma. The associations between metabolomic variables and $\alpha$ - and $\beta$-diversity indexes and relative taxa abundances were tested using multi-adjusted partial Spearman correlations, PERMANOVAs, and MaAsLins, respectively. A Multiple testing correction was applied (Benjamini-Hochberg, 10\%-FDR). Microbial richness was negatively associated with lipid-related signals and positively associated with amino acids, choline, creatinine, glucose, and citrate $(-0.133$ $\leq$ Spearman's $\rho \leq 0.126$ ). Specific associations between metabolomic signals and abundances of taxa were detected (25 at the genus level and 19 at the species level): notably, numerous associations were observed for creatinine (positively associated with 11 species, and negatively associated with Faecalibacterium prausnitzii). This large-scale population-based study highlights metabolites associated with gut microbial features and provides new insights into the understanding of complex host-gut microbiota metabolic relationships. In particular, our results support the implication of a "gut-kidney axis". More studies providing a detailed exploration of these complex interactions, and their implications for host health are needed. 


\section{INTRODUCTION}

The gut metagenome has been estimated to be 150 to 400 times larger than the human genome ${ }^{(1,2)}$, yielding a colossal metabolic potential ${ }^{(3)}$. Products of the microbial metabolism comprise a vast range of molecules involved in a variety of biological functions ${ }^{(4,5)}$ and integrated in proposed "host-microbial metabolic axes". These molecules complement the host metabolism and subsequently influence the host health through potential beneficial or harmful effects ${ }^{(5-9)}$. Consequently, the gut microbiota is now fully considered as an endocrine pseudo-organ within the mammalian holobiont superorganism ${ }^{(6,10)}$.

Specific studies investigating targeted molecules - such as short-chain fatty acids, bile acids, choline metabolites, etc. - have considerably increased the mechanistic knowledge of microbialhuman co-metabolism ${ }^{(4,11-13)}$. However, studies addressing more comprehensively the highly complex microbial-host metabolic interactions remain scarce ${ }^{(9)}$. Metabolomics - the systematic identification and quantification of the low-molecular weight metabolic products of a biological system at a specific point in time - enables the simultaneous detection and relative quantification of hundreds of molecules, and thus holds promise to elucidate microbial-host interactions ${ }^{(14)}$. Studies integrating a metabolomic approach have started to expand. Notably, gut microbiota has been reported to associate with the serum metabolome. Pedersen et al. indeed showed that the serum metabolome of insulin-resistant individuals presents increased levels of branched-chain amino acids (BCAAs), which were further correlated with gut microbial genetic characteristics (namely the potential for biosynthesis of BCAAs and lack of genes encoding bacterial inward transporters BCAAs) ${ }^{(15)}$. Additionally, comparisons of the host metabolomes (in plasma or urine) between diseased individuals ${ }^{(16-18)}$ or subjects included in nutritional interventions ${ }^{(19-22)}$ and controls have detected molecules stemming exclusively from microbial activity (e.g. hippurate or p-cresol) as discriminant metabolites. However, in these studies, neither the gut microbiota composition nor its activity were analyzed, and direct associations between the gut microbiota and the host metabolome were not investigated. In fact, research investigating such associations is scarce and often have been 
Accepted manuscript

conducted on limited sample sizes $(\mathrm{N} \leq 100)^{(9,23-27)}$, or focused on subjects with specific characteristics or conditions like chronic heart failure ${ }^{(23)}$, spleen-yang-deficiency syndrome ${ }^{(26)}$, obesity ${ }^{(24,27)}$, physiological and metabolic stress ${ }^{(25)}$, etc. More recently, studies were performed on larger population-based samples, including several hundreds of participants and reporting associations between the composition and diversity of the gut microbiota and circulating metabolites, using metabolomics ${ }^{(28-32)}$. Following these approaches, our objective was to investigate the associations between untargeted plasma metabolomic signatures and gut microbiota composition in a large sample of healthy French subjects.

\section{METHODS}

\section{Study population}

This study was conducted in the framework of the Milieu Intérieur project, which enrolled 1,000 healthy French adults with primary aim to assess the determinants of immunologic variance within the general healthy population. The objectives of the present study are secondary objectives to the Milieu Intérieur project, for which the rationale, design, and methods have been extensively described elsewhere ${ }^{(33)}$. Briefly, 500 females and 500 males equally distributed across 5 classes of age (from 20 to 69 years old), and of mainland French descent for at least 3 generations, were recruited in the suburban area of Rennes (Ille-et-Vilaine, Bretagne, France), between September 2012 and August 2013. Participants were considered "healthy" upon recruitment, as defined per stringent exclusion criteria comprising any chronic disease or condition involving the immune system, abnormal physical examination, or any abnormal clinical-biological analysis (33). Questionnaires and biological samples were administered and collected by trained medical investigators supported by a full clinical team. The study is sponsored by Institut Pasteur (Pasteur ID-RCB Number: 2012-A00238-35). It was conducted as a single center study and without any investigational product, and was approved by Comité de Protection des Personnes - Ouest 6 on 06/13/2012 (CPP Ouest 6-728/MS2) and by Agence Nationale de Sécurité du Médicament on 06/22/2012 (ID-RCB Number: 2012-A00238-35, ref. ANSM: B120239-70). The protocol, which is 
registered under ClinicalTrials.gov (NCT01699893), was designed and conducted in accordance with the Declaration of Helsinki and good clinical practice as outlined in the International Conference on Harmonization of Technical Requirements for Registration of Pharmaceuticals for Human Use Guidelines for Good Clinical Practice.

\section{Data collection}

\section{Covariates}

During the medical visit, investigators measured body-mass index (BMI) using standardized methods, and collected information related to age, sex, smoking status, educational level, employment status, occupational category, income level, and physical activity.

\section{Gut microbiota profiling from stool samples}

Gut microbiota composition was determined through the sequencing of the $16 \mathrm{~S}$ ribosomal RNA gene in stool samples produced at home maximum 24 hours before the scheduled medical visit, collected in a double-lined sealable bag maintaining strict anaerobic conditions, and aliquoted and stored at $-80^{\circ} \mathrm{C}$ upon reception at the clinical site. Detailed information regarding DNA preparation, barcoding Polymerase Chain Reaction (PCR) protocol, sequencing, and computation of microbial diversity indexes are provided in Supplementary Material and Methods 1.

\section{Untargeted NMR metabolomics analysis in plasma samples}

Fasting whole blood was collected in heparin tubes and centrifuged $(300 \mathrm{~g}, 10 \mathrm{~min}$, room temperature) maximum $6 \mathrm{~h}$ after blood draw, and supernatants were stored at $-80^{\circ} \mathrm{C}$ maximum 10min after centrifugation. Plasma samples underwent 2 freeze-thaw cycles before proton nuclear magnetic resonance (NMR) analysis, the additional freeze-thaw cycle being done for all samples in order to prepare internal quality control samples necessary for the study ${ }^{(34,35)}$ and so that that all samples would go through the same process. The NMR analysis was performed blindly on samples (randomized for age and sex) according to a previously published protocol ${ }^{(36)}$. Briefly, two complementary one-dimensional NMR sequences were acquired per plasma sample, on a 500MHzBruker Avance III spectrometer (Bruker, Billerica, MA, USA) at 300K - namely the ${ }^{1} \mathrm{H}$ 1D NMR 
Accepted manuscript

pulse sequence Nuclear Overhauser Effect Spectroscopy (NOESY1D) with z-gradient (Bruker pulse program noesygppr1d) and the Carr-Purcell-Meiboom-Gill (CPMG) sequences. Consequently, to acquisition, all spectra were pre-processed, calibrated, and ultimately "sliced" into "buckets" with pre-defined limits so as to maximize recovery of peak entities. The buckets were then scaled to the total summed integrals for each spectrum, and the integrals of peak entities were calculated to obtain continuous NMR variables that were used in further statistical analyses. Finally, NMR signals were assigned using ad hoc literature ${ }^{(37)}$. CHENOMX software (CHENOMX, Edmonton, Alberta, Canada) and Human Metabolome Database ${ }^{(38)}$ were used to confirm the assignments using the medium spectrum (Supplementary Figure 3) ${ }^{(37)}$. After the statistical analyses, the assignments of the buckets that associated with the gut microbiota were newly carefully looked and confirmed to be unambiguously assigned by gathering information from S-TOCSY, additional two-dimension NMR experiments (recorded on random samples) such as $1 \mathrm{H}-1 \mathrm{H}$ Total Correlated Spectroscopy (TOCSY) and J-resolved experiments. The detailed NMR metabolomic analysis protocol (sample preparation, post-acquisition spectra pre-processing, and intelligent bucketing of spectra and obtaining of NMR variables) is provided in Supplementary Material and Methods 2.

\section{Statistical analyses}

Associations between each NMR variable and each $\alpha$-diversity index were tested using nonparametric partial Spearman correlations. Associations between each NMR variable and interindividual dissimilarities in gut microbiota composition (Bray-Curtis index of $\beta$-diversity) were tested using permutational analysis of variance (PERMANOVA) with 999 permutations. Associations between each NMR variable and the relative abundances of genera and species were tested using multivariate associations with linear models (MaAsLin). This statistical framework performs boosted, additive general linear models and is suited to test the associations between the relative abundance of microbial community members and metadata (here the NMR variables and covariates). Relative abundances are first transformed through a variance-stabilizing arcsin-square 
root transformation. Genera and species were included only if their relative abundance and prevalence were higher than $0.01 \%$ and $1 \%$, respectively.

Partial Spearman correlations, PERMANOVAs and MaAsLins were all multi-adjusted for age (20 to $<30,30$ to $<40,40$ to $<50,50$ to $<60$, and 60 to $<70$ years old), sex, BMI (continuous variable, in kg.m ${ }^{-2}$ ), smoking status (non-smoker, ex-smoker, current smoker), and physical activity (continuous variable, in $\mathrm{h} . \mathrm{wk}^{-1}$ ). These covariates were indeed reported in the literature to have a potential impact on gut microbiota composition or metabolomic signature, or significantly associated with microbial or metabolomic features in our analyses. These associations between covariates and gut microbial and metabolomic features were tested using PERMANOVAs and Principal Component Partial R-square (PC-PR2) analysis, respectively, enabling to measure the percentage of variation in microbial composition or metabolomic data explained by the factors tested. Additionally, $\alpha$ - and $\beta$ diversity models (partial Spearman correlations and PERMANOVAs) were adjusted for sequencing depth. P-values were adjusted for multiple testing using a Benjamini-Hochberg correction with a 10\%-False Discovery Rate (FDR).

To better visualize the interindividual variation in metabolomics signatures in our study sample, Principal Component Analysis (PCA) of the metabolomic datasets were plotted and color-coded based on age, sex, and BMI.

Spearman correlations were performed using SAS 9.4; PERMANOVAs, MaAsLins, PC-PR2, and PCA representations of metabolomic datasets were performed using R 3.3.2 (packages vegan, Maaslin, FactoMineR, and FactoExtra).

\section{RESULTS}

\section{Characteristics of the study sample}

Among 1,000 participants to the Milieu Intérieur study, 138 were excluded from the study sample because of missing gut microbiota composition data - due to failure during PCR, insufficient number of detected reads, or insufficient quantity of stool aliquot or DNA extracted. Among the 862 participants with available gut microbiota data, 16 were further excluded because their 
metabolomic signatures could not be determined - due to insufficient quantity of plasma aliquot, or failure during NMR spectra acquisition. Participant flow-chart is presented in Supplementary Figure 1. Characteristics of the 846 subjects are presented in Table 1. Mean \pm standard deviation (SD) BMI was $24.3 \pm 3.3 \mathrm{~kg} \cdot \mathrm{m}^{-2}$ and mean \pm SD physical activity (including both professional and leisure physical activity) was $5.4 \pm 6.3 \mathrm{~h} . \mathrm{wk}^{-1}$. The proportion of smokers was $19.9 \%$.

Characteristics of the gut microbiota in the Milieu Intérieur study have been previously described $(39,40)$. Microbiota analysis yielded sequencing depth ranging from 5,064 to 240,472 reads per sample (mean sequencing depth \pm SD: $21,427 \pm 19,180$ reads). Detected reads clustered into 8,422 OTUs classified into 11 phyla, 328 genera, and 698 species. Firmicutes (mean relative abundance \pm SD: $0.68 \pm 0.13)$, Bacteroidetes (0.29 \pm 0.13$)$, Proteobacteria $(0.02 \pm 0.03)$, Actinobacteria $(0.007 \pm$ $0.01)$, and Verrucomicrobia $(0.004 \pm 0.008)$ were the 5 most abundant phyla. Supplementary Table 1 presents the 22 genera constituting the "core microbiota" - defined as the genera shared by at least $95 \%$ of samples ${ }^{(41)}$ - in our study population. A complete description of genera and species composing the gut microbiota of our sample is available in Supplementary Table 2. Regarding $\alpha$ diversity indexes, Simpson's index ranged from 0.41 to 0.98 (mean \pm SD: $0.92 \pm 0.05$ ); observed richness from 56 to 346 (mean \pm SD: $193.4 \pm 55.1$ ) and Chao1 richness estimate from 62 to 1039 (mean \pm SD: $298.3 \pm 117.0$ ). Univariate associations measuring the percentage of variation in interindividual dissimilarities in the gut microbial composition (Bray-Curtis index of $\beta$-diversity) that is explained by different covariates (age, sex, BMI, smoking status, physical activity, and sequencing depth) are shown in Supplementary Table 3.

NMR analysis on plasma samples generated 260 CPMG and 269 NOESY1D variables. Spectral regions most likely corresponding to noise (i.e. CPMG regions 0-0.5ppm, 4.3-5.0ppm, and 8.5510.0ppm; and NOESY1D regions 4.3-5.0ppm, and 9.5-11.0ppm) were excluded from further analysis. A list of the resulting 424 NMR continuous variables used in our analyses (202 CPMG signals and 215 NOESY1D: spectral regions with their metabolite assignments, as well as mean and SD) is presented in Supplementary Tables 4 and 5. Results of the PCA performed on the CPMG 
Accepted manuscript

and NOESY1D metabolomic datasets are shown in Figure 1 and Supplementary Figure 2, respectively. Although no clear clustering appears, grouping patterns along gradient of sex, age, and BMI can be observed. Results of the PC-PR2 analysis measuring the percentage of variation in the metabolomic dataset that is explained by the covariates (age, sex, BMI, smoking status, and physical activity) are shown in Supplementary Table 6.

\section{Associations between plasma metabolomic signals and $\alpha$ - and $\beta$-diversity indexes}

The associations between metabolomic variables and $\alpha$-diversity indexes are presented in Figure 2 . Overall, and after correcting for multiple testing with a 10\%-FDR, observed richness was associated with 24 CMPG and 8 NOESY1D signals. Negative associations were observed with lipid-related signals (namely lipoproteins or unsaturated lipids) as well as with ester- and ketone-related metabolites (partial Spearman's $\rho$ range: -0.133 to -0.097 ), while positive associations were detected with signals pertaining to amino acids (polar glutamine and histidine and aromatic tyrosine, as well as related metabolites) and proteins, creatinine, as well as with choline, and glycolysis-related metabolites (glucose and citrate) (partial Spearman's $\rho$ range: 0.087 to 0.126 ). Similar results were obtained with Chaol richness estimate but no association was detected with Simpson's $\alpha$-diversity index. Likewise, no association was observed between NMR metabolomic variables and inter-individual dissimilarities in gut microbiota composition (Bray-Curtis $\beta$-diversity index).

\section{Associations between plasma metabolomic signals and bacterial taxa (relative abundances)}

The associations (significant at 10\%-FDR) between NMR metabolomic variable and the relative abundance of gut microbiota genera and species are presented in Table 2. At the genus and species levels, 25 and 19 associations were detected, respectively. Associated taxa comprised genera and species with prevalence ranging from $20 \%$ to $100 \%$ of the study population. Creatinine was the metabolite associated with most taxa (16 genera and 11 species). Notably, consistent positive associations were found between the CPMG and the NOESY1D analyses for genera Catabacter, 
Accepted manuscript

Saccharofermentans, and Oscillibacter and a negative association was observed for Faecalibacterium prausnitzii.

\section{DISCUSSION}

In the present work, we investigated the associations between characteristics of the gut microbiota namely $\alpha$-diversity, inter-individual dissimilarities, and the relative abundances of genera and species - and plasma NMR metabolomic signatures of the host in a large population-based sample of 846 healthy French adults. Overall, our results suggest that gut bacterial features are associated with the systemic metabolism of the host. In particular, we found that bacterial richness was positively associated with signals pertaining to amino acids (glutamine, histidine, tyrosine), as well as with creatinine, choline and glycolysis-related metabolites, while negative associations were observed with lipid-, as well as ester-, and ketone-related signals. Finally, specific associations between metabolomic signals and bacterial members of the gut microbiota were detected.

The metabolic relevance and importance of the gut microbiota for the host are now widely admitted, and research scrutinizing host-gut microbial metabolic relationships is an active area of investigation ${ }^{(42)}$. However, studies investigating direct associations between gut microbiota composition and host blood metabolome in large population-based samples are scarce while more available studies have been conducted on a limited number of participants or focused on specific populations ${ }^{(15,23,26,27)}$. In particular, a study by Org et al. assessed the relationships between gut microbiota composition and circulating metabolites in 531 healthy Finnish males from the METSIM cohort ${ }^{(28)}$. Consistently with their study, we reported positive correlations between bacterial richness and glutamine, histidine, and creatinine - with similar Spearman correlation coefficients. Furthermore, a study on 399 subscribers to a US Scientific Wellness program was able to explain $45 \%$ of the gut microbiota alpha-diversity with 40 plasma metabolites, among which a high frequency of phenylalanine and tyrosine metabolites (including p-cresol sulfate, a potentially uremic toxin) and hippurate ${ }^{(32)}$ while a study in 1529 females from the TwinsUK cohort found five metabolites that consistently correlated with gut microbiota alpha-diversity (Shannon index) 
Accepted manuscript

including hippurate, p-cresol sulfate, phenylacetylglutamine, 3-phenylpropionate, and hyodeoxycholate ${ }^{(31)}$.

In a study conducted on 893 participants from the Dutch LifeLines-DEEP cohort, Fu et al. ${ }^{(13)}$ measured specific lipid levels through targeted colorimetric methods, and found that triglycerides and high-density lipoproteins were respectively negatively and positively associated with both bacterial richness and diversity. Consistently, a more recent study using NMR metabolomics in 2309 individuals from the Rotterdam Study and the LifeLines-DEEP cohort observed inverse associations between the diversity of the gut microbiota and serum levels of VLDL particles, triglycerides, total fatty acids, monounsaturated fatty acids and saturated fatty acids but also glycoprotein acetyl, alanine, isoleucine, and lactate and positive associations with HDL particles. In addition, Le Chatelier et al. associated a decreased gut microbial richness with metabolic impairments such as increased fat mass and body weight, inflammation of the adipose tissue and dyslipidemia ${ }^{(43)}$. In the present work, we consistently found that lipid-related metabolomic signals were negatively associated with bacterial richness. However, we were unable to further characterize these lipid-related signals and discriminate between the different lipid classes potentially hampering the detection of specific associations previously reported ${ }^{(13,28)}$. Indeed, long-chain metabolites such as fatty acids result in an overlap of signals of protons in NMR, limiting a more specific assignment ${ }^{(44)}$. Still, it should be noted that, in our sample, NMR signals assigned to lipids demonstrated excellent correlation with blood triglycerides (Spearman's $\rho>0.65$, p-value $<0.0001$ for spectral regions $1.207-1.311 \mathrm{ppm}, 2.151-2.166 \mathrm{ppm}$, and 2.166-2.167ppm), adding support to the consistency of our results with those previous findings. Furthermore, high-fat Western diet has consistently been associated with gut microbiota impairments ${ }^{(45,46)}$, and lipid-related signals in our study correlated positively with dietary intakes of processed meat (Spearman's $\rho=0.09$, p-value $=0.006$ for spectral regions $2.166-2.167 \mathrm{ppm}$ and $\rho=0.08$, $\mathrm{p}$-value $=0.02$ for $1.207-1.311 \mathrm{ppm}$ and $2.151-2.166 \mathrm{ppm}$ ) and fried products $(\rho=0.07$, $p$-value $=0.04$ for $2.166-2.167 \mathrm{ppm})$, and negatively correlated with fruit $(\rho=$ $-0.09, \mathrm{p}$-value $=0.01$ for $1.207-1.311 \mathrm{ppm}$ and $2.166-2.167 \mathrm{ppm}$ and $\rho=-0.08, \mathrm{p}$-value $=0.02$ for 2.151 - 
Accepted manuscript

$2.166 \mathrm{ppm})$ and vegetables $(\rho=-0.09$, $\mathrm{p}$-value $=0.007$ for $2.166-2.167 \mathrm{ppm}$ and $\rho=-0.07$, $\mathrm{p}$ value $=0.04$ for $2.151-2.166 \mathrm{ppm})$. Nonetheless, these assumptions should be considered with caution as additional exploration and targeted identification of these lipid signals using mass spectrometry (MS) or lipidomics is necessary to get more detailed insights into the associations we observed.

Our finding of a positive association between glutamine and gut bacterial richness echoes a result from De Souza et al. who suggested that glutamine might have an anti-inflammatory effect through a modulation of specific gut bacterial members following the observation that an oral supplementation with L-glutamine decreased the Firmicutes/Bacteroidetes ratio in obese and overweight adults ${ }^{(47)}$.

In our study, creatinine was the metabolomic signal most associated with the relative abundances of bacterial taxa. This by-product of muscle metabolism is exclusively excreted through the kidneys and a high creatinine level in blood is notably used as an indicator of kidney dysfunction ${ }^{(48)}$. Our finding of positive associations between plasma creatinine and the relative abundance of various representatives of the Clostridiales order and the Ruminococcaceae family matches results from Org et al. ${ }^{(28)}$. We also reported a negative association between creatinine and Faecalibacterium prausnitzii, a species drawing a particular attention from the research community as it has been shown to exert anti-inflammatory properties through its ability to produce anti-inflammatory metabolites (e.g. butyrate), or to inhibit of the production of pro-inflammatory cytokines ${ }^{(49)}$. Various studies have reported that $F$. prausnitzii is reduced in conditions such as ulcerative colitis, inflammatory bowel disease, colorectal cancer, diabetes, psoriasis, etc. ${ }^{(49-51)}$. Consequently, this species has been proposed as a potential biomarker of gut health ${ }^{(52)}$ and is even considered as an interesting probiotic candidate ${ }^{(53)}$. In particular, a decrease in $F$. praunitzii was previously reported in chronic kidney disease patients compared to healthy controls ${ }^{(54,55)}$. This echoes our result of a negative association between $F$. praunitzii and creatinine obtained in a healthy sample without renal dysfunction. Overall, the numerous associations we detected between bacterial taxa and creatinine are in support of a gut-kidney conversation ${ }^{(56,57)}$. In this previously described bi-directional organ 
axis, gut contributes to inflammation and renal injury through microbiota dysbiosis and dysregulations in microbial metabolite production. Conversely, kidney perturbations lead to dysbiosis and disruptions in tight junction function therefore promoting inflammatory conditions in the gut. Notably, Jiang et al. reported that indicators of the renal function such as serum creatinine, estimated glomerular filtration rate, and cystatin $\mathrm{C}$ were the most important environmental parameters to influence the overall microbial communities in end-stage renal disease patients ${ }^{(55)}$. In our study a link between the gut and the kidneys seemed to be already observed even in a healthy setting where individuals were free of kidney or gut impairments. However, further exploration of renal function indicators (e.g. estimated glomerular filtration rate, cystatin $\mathrm{C}$, and urine creatinine) would be of utmost importance to confirm such a link. If we hypothesize a gut-kidney axis to interpret our results, alternate interpretations could also be proposed. Indeed, negative associations have also been reported between trymethylamine N-oxide (TMAO) and F. prausnitzii ${ }^{(28,58)}$ and creatinine is a precursor for the gut bacterial synthesis of trimethylamine (TMA), which is further oxidized into TMAO in the liver ${ }^{(48)}$. Therefore, we cannot rule out that the association we observed between creatinine and $F$. prausnitzii may actually reflect the overall TMAO metabolic pathway instead of being a true association. However, because TMAO could not be detected by NMR in our study, it was not possible to verify this assumption.

An important strength of this work pertains to its design - namely the acquisition of both detailed metabolomics data and gut microbiome data in a large population-based sample of healthy adults. While individual or specific approaches consider biomolecules separately, metabolomics allows for the simultaneous detection and relative quantification of hundreds of molecules hence the monitoring of subtle system-wide metabolic interactions ${ }^{(59)}$. In this study we used NMRmetabolomics with standardized and optimized protocols yielding high-quality data ${ }^{(60)}$. This robust and stable technology has demonstrated its suitability for long-term epidemiological studies ${ }^{(61)}$, notably by allowing to analyze large samples quickly with a high reproducibility ${ }^{(62)}$. From a statistical standpoint, the analyses implemented here allowed to explore detailed associations 
between gut bacterial and metabolomic datasets, beyond the sole assessment of global connections obtained through multivariate correlation-based approaches (e.g. co-inertia or Procrustes analyses). In addition, we were able to take into account a range of factors reported to influence the gut microbiota composition or the systemic metabolism of the host in our models, thus mitigating potential confounding. Nonetheless, residual confounding cannot be ruled out. For instance, intestinal transit time was shown to be a major confounding factor of gut microbiota composition ${ }^{(63)}$, and we were not able to collect this information. Another important strength and originality of this work is that it was based on a large sample from the general population including only healthy men and women selected using strict criteria. Hence, our results were not confounded by underlying disease and reflected normal range. Yet, this may also have induced less variations in plasma metabolites levels or in gut microbiota composition and therefore resulted in associations of smaller magnitude. Some limitations should also be acknowledged for this study. First, the cross-sectional design of our study, in which blood and stool samples were taken at the same time, prevents from drawing any conclusion regarding the sequential causality of events between host metabolism (e.g., creatinine levels) and gut microbiota composition (e.g., F. prausnitzii). Further, plasma metabolomic signatures only give a metabolic "snapshot" that results from both the endogenous and the gut microbial metabolisms, which makes it is not possible to discriminate signals from human or microbial origin. In future studies, subsequent time points with both metabolomic and microbiota data collected would be of utmost interest to investigate the stability over time and the chronology of the associations observed in the present cross-sectional work. In addition, the fact that most detected metabolites result from multiple metabolic pathways that may be related to gut microbiota or not may also explain the modest correlation coefficients observed between plasma metabolites and gut microbial richness. It should also be acknowledged that, although highly informative, the use of 16S rRNA gene sequencing and NMR metabolomics presents technical limitations. Shotgun metagenomics for instance would give a more precise characterization of microbiota features and also bring additional information regarding the functional metabolic capacity of the gut microbiota. 
This would allow to go beyond the compositional approach, limited by the redundancy of gene pool and metabolic functionalities between distinct gut microbial members ${ }^{(64,65)}$, but still relevant according to recent studies arguing that some metabolic pathways pertain to a handful of species ${ }^{(66)}$. Likewise, while NMR detects only the most abundant metabolites ( $\mu \mathrm{M}$ to $\mathrm{mM}$ range), mass spectrometry (MS) has lower detection limits and higher sensitivity for signal assignment, and would therefore allow the detection of metabolites undetectable with NMR ${ }^{(67)}$. This technical limitation of NMR may explain why several low-concentration metabolites deriving specifically from gut microbiota were not detected in our study. As already mentioned, the precision of NMR bucket assignment was also limited for some metabolites, especially for lipids and proteins. For instance, the unsaturation corresponds to a chemical function in the aliphatic chain of various lipids, and it is impossible to differentiate among the different lipid types within the plasma sample. Hence, this limits the comparability of our results to prior studies. In addition, plasma samples underwent two freeze-thaw cycles prior to NMR analyses (necessary to prepare internal quality controls) so that the possibility of increased metabolites cleavage cannot be ruled out. Still, this would similarly affect all samples as they all went through the same process.

Although the Milieu Intérieur population is somewhat representative of the French Ille-et-Vilaine region ${ }^{(33)}$, our study sample is not representative of the whole French adult population - caution is therefore needed in the generalization of our results. However, it is important to acknowledge that from a gut microbiota composition standpoint, our study sample was consistent with other studies performed in Western settings ${ }^{(41,68)}$, with the same 5 most abundant phyla and shared "core genera" (Supplementary Table 1). Finally, the results highlighted here were mostly exploratory as they were obtained from a single population. These results will need to be replicated and confirmed in the future, using independent samples and/or analytical techniques allowing a better refinement of the detected metabolites (e.g., MS, lipidomics). In addition, the hypotheses we proposed to interpret our results were based on existing literature and would need to be developed and confirmed in experimental settings in vitro and/or in vivo. In particular, detailed mechanistic studies 
Accepted manuscript

comprehensively elucidating the metabolic capability of gut microorganisms are needed to enlighten the associations we detected.

Overall, the present study provides interesting insights regarding the associations between gut microbiota composition and plasma metabolic signatures of the host. Our results contribute to a better understanding of the host-gut microbiota relationships, and notably highlight a possible gutkidney axis in healthy subjects. Because the associations we found in healthy individuals were already reported in chronic kidney disease patients, this builds up to the concept that onset of chronic diseases is a lengthy process that could be detected at early stages. This appears all the more interesting that microbial-host co-metabolites were shown to be excellent prodromal markers of future divergence in metabolic and behavioral outcomes in mice ${ }^{(69)}$. However, our results remain to be confirmed in independent populations, underlying mechanisms to be elucidated, and causal implications for the host health to be established.

Acknowledgements: The authors wish to thank École doctorale 474 Frontières du Vivant Programme Bettencourt for the financial support provided to VP's Ph.D. The Milieu Intérieur ConsortiumII: Laurent ABEL (Hôpital Necker); Andres ALCOVER; Hugues ASCHARD; Kalla ASTROM (Lund University); Philippe BOUSSO; Pierre BRUHNS; Ana CUMANO; Caroline DEMANGEL; Ludovic DERIANO; James DI SANTO; Françoise DROMER; Gérard EBERL; Jost ENNINGA; Jacques FELLAY (EPFL, Lausanne); Odile GELPI; Ivo GOMPERTS-BONECA; Milena HASAN; Serge HERCBERG (Université Sorbonne Paris Nord); Olivier LANTZ (Institut Curie); Claude LECLERC; Hugo MOUQUET; Etienne PATIN; Sandra PELLEGRINI; Stanislas POL (Hôpital Cochin); Antonio RAUSELL (INSERM U1163 - Institut Imagine); Lars ROGGE; Anavaj SAKUNTABHAI; Olivier SCHWARTZ; Benno SCHWIKOWSKI; Spencer SHORTE; Vassili SOUMELIS (Institut Curie); Frédéric TANGY; Eric TARTOUR (Hôpital Européen Georges Pompidou); Antoine TOUBERT (Hôpital Saint-Louis); Mathilde TOUVIER (Université Sorbonne Paris Nord); Marie-Noëlle UNGEHEUER; Matthew L. ALBERT (Roche Genentech)§; 
Accepted manuscript

Darragh DUFFY§; Lluis QUINTANA-MURCI§. II Unless otherwise indicated, partners are located at Institut Pasteur, Paris, France. § Coordinator of the Milieu Intérieur Consortium. Additional information can be found at: http://www.milieuinterieur.fr/en

Financial support: This work was supported by French Government's Investissement d'Avenir Program, Laboratoire d'Excellence “Milieu Intérieur" Grant ANR-10-LABX-69-01. The funding body had no role in the design of the study, the collection, analysis, and interpretation of data, or in the writing of the manuscript.

Conflict of interest: The authors declare that they have no competing interests.

Authors' contributions: VP, MT: designed the research; LQM, MLA, DD, OL, MT, MI consortium: conducted the research; SM: performed microbiota sequencing; VP: performed metabolomic signature acquisition; NB, PS, MNT: supervised metabolomic signatures acquisition; VP: performed statistical analysis; MT: supervised statistical analysis; VP: wrote the original draft; MDT: revised the manuscript; VP, MDT, SM, AVB, ND, LL, CBD, MJT, EKG, BC, EP, KEA, PLM, CJ, PG, SH, LQM, MLA, DD, OL, PS, MNT, MT, MI Consortium: contributed to the data interpretation and revised each draft for important intellectual content; MT had primary responsibility for final content; and LQM, MLA, DD are coordinators of the MI Consortium. All authors read and approved the final manuscript. 


\section{REFERENCES}

1. MetaHIT Consortium, Qin J, Li R, et al. (2010) A human gut microbial gene catalogue established by metagenomic sequencing. Nature 464, 59-65.

2. MetaHIT Consortium, Li J, Jia H, et al. (2014) An integrated catalog of reference genes in the human gut microbiome. Nature Biotechnology 32, 834-841.

3. Yadav M, Verma MK \& Chauhan NS (2018) A review of metabolic potential of human gut microbiome in human nutrition. Archives of Microbiology 200, 203-217.

4. Nicholson JK, Holmes E, Kinross J, et al. (2012) Host-Gut Microbiota Metabolic Interactions. Science 336, 1262-1267.

5. Schroeder BO \& Bäckhed F (2016) Signals from the gut microbiota to distant organs in physiology and disease. Nature Medicine 22, 1079-1089.

6. Brown JM \& Hazen SL (2015) The Gut Microbial Endocrine Organ: Bacterially Derived Signals Driving Cardiometabolic Diseases. Annual Review of Medicine 66, 343-359.

7. Rajani C \& Jia W (2018) Disruptions in gut microbial-host co-metabolism and the development of metabolic disorders. Clinical Science 132, 791-811.

8. Postler TS \& Ghosh S (2017) Understanding the Holobiont: How Microbial Metabolites Affect Human Health and Shape the Immune System. Cell Metabolism 26, 110-130.

9. Li M, Wang B, Zhang M, et al. (2008) Symbiotic gut microbes modulate human metabolic phenotypes. Proceedings of the National Academy of Sciences 105, 2117-2122.

10. Kundu P, Blacher E, Elinav E, et al. (2017) Our Gut Microbiome: The Evolving Inner Self. Cell 171, 1481-1493.

11. Louis P, Hold GL \& Flint HJ (2014) The gut microbiota, bacterial metabolites and colorectal cancer. Nature Reviews Microbiology 12, 661-672.

12. Ryan PM, Stanton C \& Caplice NM (2017) Bile acids at the cross-roads of gut microbiome-host cardiometabolic interactions. Diabetology \& Metabolic Syndrome 9.

13. Fu J, Bonder MJ, Cenit MC, et al. (2015) The Gut Microbiome Contributes to a Substantial Proportion of the Variation in Blood Lipids. Circulation Research 117, 817-824. 
14. Smirnov KS, Maier TV, Walker A, et al. (2016) Challenges of metabolomics in human gut microbiota research. International Journal of Medical Microbiology 306, 266-279.

15. Pedersen HK, Gudmundsdottir V, Nielsen HB, et al. (2016) Human gut microbes impact host serum metabolome and insulin sensitivity. Nature 535, 376-381.

16. Kelly RS, Sordillo JE, Lasky-Su J, et al. (2018) Plasma metabolite profiles in children with current asthma. Clinical \& Experimental Allergy 48, 1297-1304.

17. Naviaux RK, Naviaux JC, Li K, et al. (2016) Metabolic features of chronic fatigue syndrome. Proceedings of the National Academy of Sciences 113, E5472-E5480.

18. Urpi-Sarda M, Almanza-Aguilera E, Llorach R, et al. (2019) Non-targeted metabolomic biomarkers and metabotypes of type 2 diabetes: A cross-sectional study of PREDIMED trial participants. Diabetes \& Metabolism 45, 167-174.

19. Derkach A, Sampson J, Joseph J, et al. (2017) Effects of dietary sodium on metabolites: the Dietary Approaches to Stop Hypertension (DASH)-Sodium Feeding Study. The American Journal of Clinical Nutrition 106, 1131-1141.

20. Mora-Cubillos X, Tulipani S, Garcia-Aloy M, et al. (2015) Plasma metabolomic biomarkers of mixed nuts exposure inversely correlate with severity of metabolic syndrome. Molecular Nutrition \& Food Research 59, 2480-2490.

21. Pantophlet AJ, Wopereis S, Eelderink C, et al. (2017) Metabolic Profiling Reveals Differences in Plasma Concentrations of Arabinose and Xylose after Consumption of Fiber-Rich Pasta and Wheat Bread with Differential Rates of Systemic Appearance of Exogenous Glucose in Healthy Men. The Journal of Nutrition 147, 152-160.

22. Tovar J, de Mello VD, Nilsson A, et al. (2017) Reduction in cardiometabolic risk factors by a multifunctional diet is mediated via several branches of metabolism as evidenced by nontargeted metabolite profiling approach. Molecular Nutrition \& Food Research 61, 1600552.

23. Cui X, Ye L, Li J, et al. (2018) Metagenomic and metabolomic analyses unveil dysbiosis of gut microbiota in chronic heart failure patients. Scientific Reports $\mathbf{8}$.

24. Dewulf EM, Cani PD, Claus SP, et al. (2013) Insight into the prebiotic concept: lessons from an exploratory, double blind intervention study with inulin-type fructans in obese women. Gut 62, 11121121. 
Accepted manuscript

25. Karl JP, Margolis LM, Madslien EH, et al. (2017) Changes in intestinal microbiota composition and metabolism coincide with increased intestinal permeability in young adults under prolonged physiological stress. American Journal of Physiology-Gastrointestinal and Liver Physiology 312, G559G571.

26. Lin Z, Ye W, Zu X, et al. (2018) Integrative metabolic and microbial profiling on patients with Spleenyang-deficiency syndrome. Scientific Reports 8.

27. Liu R, Hong J, Xu X, et al. (2017) Gut microbiome and serum metabolome alterations in obesity and after weight-loss intervention. Nature Medicine 23, 859-868.

28. Org E, Blum Y, Kasela S, et al. (2017) Relationships between gut microbiota, plasma metabolites, and metabolic syndrome traits in the METSIM cohort. Genome Biology 18.

29. Vojinovic D, Radjabzadeh D, Kurilshikov A, et al. (2019) Relationship between gut microbiota and circulating metabolites in population-based cohorts. Nat Commun 10, 5813.

30. Menni C, Zhu J, Le Roy $\mathrm{Cl}$, et al. (2020) Serum metabolites reflecting gut microbiome alpha diversity predict type 2 diabetes. Gut Microbes 11, 1632-1642.

31. Pallister T, Jackson MA, Martin TC, et al. (2017) Hippurate as a metabolomic marker of gut microbiome diversity: Modulation by diet and relationship to metabolic syndrome. Sci Rep 7, 13670.

32. Wilmanski T, Rappaport N, Earls JC, et al. (2019) Blood metabolome predicts gut microbiome $\alpha$ diversity in humans. Nat Biotechnol 37, 1217-1228.

33. Thomas S, Rouilly V, Patin E, et al. (2015) The Milieu Intérieur study - An integrative approach for study of human immunological variance. Clinical Immunology 157, 277-293.

34. Gika HG, Zisi C, Theodoridis G, et al. (2016) Protocol for quality control in metabolic profiling of biological fluids by U(H)PLC-MS. Journal of Chromatography B 1008, 15-25.

35. Dunn WB, Broadhurst D, Begley P, et al. (2011) Procedures for large-scale metabolic profiling of serum and plasma using gas chromatography and liquid chromatography coupled to mass spectrometry. Nat Protoc 6, 1060-1083.

36. Lécuyer L, Victor Bala A, Deschasaux M, et al. (2018) NMR metabolomic signatures reveal predictive plasma metabolites associated with long-term risk of developing breast cancer. International Journal of Epidemiology 47, 484-494. 
37. Nicholson JK, Foxall PJD, Spraul Manfred, et al. (1995) $750 \mathrm{MHz} 1 \mathrm{H}$ and $1 \mathrm{H}-13 \mathrm{C}$ NMR Spectroscopy of Human Blood Plasma. Analytical Chemistry 67, 793-811.

38. Wishart DS, Jewison T, Guo AC, et al. (2012) HMDB 3.0-The Human Metabolome Database in 2013. Nucleic Acids Research 41, D801-D807.

39. The Milieu Intérieur Consortium, Scepanovic P, Hodel F, et al. (2019) A comprehensive assessment of demographic, environmental, and host genetic associations with gut microbiome diversity in healthy individuals. Microbiome 7.

40. Partula V, Mondot S, Torres MJ, et al. (2019) Associations between usual diet and gut microbiota composition: results from the Milieu Intérieur cross-sectional study. The American Journal of Clinical Nutrition 109, 1472-1483.

41. Falony G, Joossens M, Vieira-Silva S, et al. (2016) Population-level analysis of gut microbiome variation. Science 352, 560-564.

42. Daliri EB-M, Wei S, Oh DH, et al. (2017) The human microbiome and metabolomics: Current concepts and applications. Critical Reviews in Food Science and Nutrition 57, 3565-3576.

43. MetaHIT consortium, Le Chatelier E, Nielsen T, et al. (2013) Richness of human gut microbiome correlates with metabolic markers. Nature 500, 541-546.

44. Khoury S, Canlet C, Lacroix M, et al. (2018) Quantification of Lipids: Model, Reality, and Compromise. Biomolecules 8, 174.

45. Davis SC, Yadav JS, Barrow SD, et al. (2017) Gut microbiome diversity influenced more by the Westernized dietary regime than the body mass index as assessed using effect size statistic. MicrobiologyOpen 6, e00476.

46. De Filippo C, Cavalieri D, Di Paola M, et al. (2010) Impact of diet in shaping gut microbiota revealed by a comparative study in children from Europe and rural Africa. Proc. Natl. Acad. Sci. U.S.A. 107, 1469114696.

47. Zambom de Souza AZ, Zambom AZ, Abboud KY, et al. (2015) Oral supplementation with I-glutamine alters gut microbiota of obese and overweight adults: A pilot study. Nutrition 31, 884-889.

48. Chhibber-Goel J, Singhal V, Parakh N, et al. (2017) The Metabolite Trimethylamine-N-Oxide is an Emergent Biomarker of Human Health. Current Medicinal Chemistry 24. 
49. Ferreira-Halder CV, Faria AV de S \& Andrade SS (2017) Action and function of Faecalibacterium prausnitzii in health and disease. Best Practice \& Research Clinical Gastroenterology 31, 643-648.

50. Sokol H, Pigneur B, Watterlot L, et al. (2008) Faecalibacterium prausnitzii is an anti-inflammatory commensal bacterium identified by gut microbiota analysis of Crohn disease patients. Proceedings of the National Academy of Sciences 105, 16731-16736.

51. Machiels K, Joossens M, Sabino J, et al. (2014) A decrease of the butyrate-producing species Roseburia hominis and Faecalibacterium prausnitzii defines dysbiosis in patients with ulcerative colitis. Gut $63,1275-1283$.

52. Lopez-Siles M, Duncan SH, Garcia-Gil L, et al. (2017) Faecalibacterium prausnitzii: from microbiology to diagnostics and prognostics. The ISME Journal 11, 841-852.

53. Martín R, Miquel S, Benevides L, et al. (2017) Functional Characterization of Novel Faecalibacterium prausnitzii Strains Isolated from Healthy Volunteers: A Step Forward in the Use of F. prausnitzii as a Next-Generation Probiotic. Frontiers in Microbiology 8.

54. Jiang S, Xie S, Lv D, et al. (2016) A reduction in the butyrate producing species Roseburia spp. and Faecalibacterium prausnitzii is associated with chronic kidney disease progression. Antonie van Leeuwenhoek 109, 1389-1396.

55. Jiang S, Xie S, Lv D, et al. (2017) Alteration of the gut microbiota in Chinese population with chronic kidney disease. Scientific Reports 7.

56. Chen Y-Y, Chen D-Q, Chen L, et al. (2019) Microbiome-metabolome reveals the contribution of gutkidney axis on kidney disease. Journal of Translational Medicine 17.

57. Evenepoel P, Poesen R \& Meijers B (2017) The gut-kidney axis. Pediatric Nephrology 32, 2005-2014.

58. Sanchez-Alcoholado L, Castellano-Castillo D, Jordán-Martínez L, et al. (2017) Role of Gut Microbiota on Cardio-Metabolic Parameters and Immunity in Coronary Artery Disease Patients with and without Type-2 Diabetes Mellitus. Frontiers in Microbiology 8.

59. Patti GJ, Yanes O \& Siuzdak G (2012) Metabolomics: the apogee of the omics trilogy. Nature Reviews Molecular Cell Biology 13, 263-269.

60. Beckonert O, Keun HC, Ebbels TMD, et al. (2007) Metabolic profiling, metabolomic and metabonomic procedures for NMR spectroscopy of urine, plasma, serum and tissue extracts. Nature Protocols $\mathbf{2}$, 2692-2703. 
Accepted manuscript

61. Dumas M-E, Maibaum EC, Teague C, et al. (2006) Assessment of Analytical Reproducibility of ${ }^{1} \mathrm{H}$ NMR Spectroscopy Based Metabonomics for Large-Scale Epidemiological Research: the INTERMAP Study. Analytical Chemistry 78, 2199-2208.

62. Emwas A-H, Roy R, McKay RT, et al. (2019) NMR Spectroscopy for Metabolomics Research. Metabolites 9, 123.

63. Roager HM, Hansen LBS, Bahl MI, et al. (2016) Colonic transit time is related to bacterial metabolism and mucosal turnover in the gut. Nature Microbiology 1.

64. Abubucker S, Segata N, Goll J, et al. (2012) Metabolic Reconstruction for Metagenomic Data and Its Application to the Human Microbiome. PLoS Computational Biology 8, e1002358 [Eisen JA, editor].

65. Holmes E, Li JV, Marchesi JR, et al. (2012) Gut Microbiota Composition and Activity in Relation to Host Metabolic Phenotype and Disease Risk. Cell Metabolism 16, 559-564.

66. Morrison DJ \& Preston T (2016) Formation of short chain fatty acids by the gut microbiota and their impact on human metabolism. Gut Microbes 7, 189-200.

67. Riekeberg E \& Powers R (2017) New frontiers in metabolomics: from measurement to insight. F1000Research 6, 1148.

68. Rothschild D, Weissbrod O, Barkan E, et al. (2018) Environment dominates over host genetics in shaping human gut microbiota. Nature $555,210-215$.

69. Dumas M-E, Rothwell AR, Hoyles L, et al. (2017) Microbial-Host Co-metabolites Are Prodromal Markers Predicting Phenotypic Heterogeneity in Behavior, Obesity, and Impaired Glucose Tolerance. Cell Reports 20, 136-148. 
Table 1. Characteristics of the study population, Milieu Intérieur study, France, 2012 (N=846).

Sex

Male

Female

$413(48.8)$

Age

$20-29$ years old

$159(18.8)$

$30-39$ years old

$165(19.5)$

$40-49$ years old

$174(20.6)$

$50-59$ years old

$171(20.2)$

$60-69$ years old

177 (20.9)

Smoking status

Non-smoker

$442(52.2)$

Ex-smoker

$236(27.9)$

Current smoker

$168(19.9)$

BMI, kg. $\mathrm{m}^{-2}$

$24.3 \pm 3.3$

Physical activity, h.wk ${ }^{-1}$

$5.4 \pm 6.3$

Simpson's index

$0.92 \pm 0.05$

Observed richness

$193.4 \pm 55.1$

Chao1 richness estimate

$298.3 \pm 117.0$

Values are $\mathrm{N}(\%)$ or mean \pm SD 
Table 2. Associations between NMR variables and relative abundances of taxa with a $Q$-value $\leq 0.1$ after multiple-testing correction, using

MaAsLins, Milieu Intérieur study, France, 2012 (N=846).

\begin{tabular}{|c|c|c|c|c|c|c|c|c|c|}
\hline & Shift (ppm) & Signal assignation & Phylum| Class $\mid$ Order| Family| & Genus & Species & $\begin{array}{l}\text { MaAsLin } \\
\text { coefficient }\end{array}$ & Coverage $^{1}$ & $\begin{array}{c}\text { p- } \\
\text { value }^{2}\end{array}$ & $\begin{array}{c}\text { Q- } \\
\text { value }^{3}\end{array}$ \\
\hline \multirow{34}{*}{ 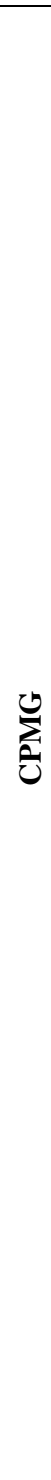 } & $1.021-1.051$ & Unassigned signal & Firmicutes| Negativicutes| Selenomonadales| Veillonellaceae| & Sporomusa* & & -68.5 & 35.22 & $<0.0001$ & 0.07 \\
\hline & $1.527-1.631$ & $\mathrm{CH}_{2} \mathrm{CH}_{2} \mathrm{COOC}$ & $\begin{array}{l}\text { Firmicutes | Clostridia| Clostridiales| Lachnospiraceae| Clostridium } \\
\text { XIVa| }\end{array}$ & Clostridium & C. bolteae & 74.2 & 99.17 & $<0.0001$ & 0.06 \\
\hline & $2.831-2.848$ & Citrate $/=\mathrm{CH}-\mathrm{CH}_{2}-\mathrm{CH}=$ & Firmicutes| Bacilli| Bacillales| Bacillaceae 1| & Caldibacillus & & -198.2 & 35.34 & $<0.0001$ & 0.07 \\
\hline & $2.967-3.071$ & Creatine/albumin lysyl & Firmicutes | Clostridia| Clostridiales| Ruminococcaceae| & Hydrogenoanaerobacterium & H. saccharovorans & -31.8 & 90.07 & $<0.0001$ & 0.07 \\
\hline & \multirow{24}{*}{ 3.071-3.111 } & \multirow{24}{*}{ Creatinine } & Firmicutes| Clostridia| Clostridiales| Ruminococcaceae| & Faecalibacterium & & -777.4 & 99.41 & $<0.0001$ & 0.02 \\
\hline & & & Firmicutes| Clostridia| Clostridiales| Ruminococcaceae| & Faecalibacterium & F. prausnitzii & -797.3 & 99.41 & $<0.0001$ & 0.03 \\
\hline & & & Firmicutes| Negativicutes| Selenomonadales| Veillonellaceae| & Sporomusa* & & 31.8 & 35.22 & $<0.0001$ & 0.06 \\
\hline & & & Firmicutes| Negativicutes| Selenomonadales| Veillonellaceae| & Sporomusa & S. termitida & 31.5 & 34.75 & $<0.0001$ & 0.06 \\
\hline & & & Tenericutes| Mollicutes| Acholeplasmatales| Acholeplasmataceae| & Acholeplasma & & 42.0 & 32.98 & 0.0001 & 0.09 \\
\hline & & & Firmicutes|Clostridia| Clostridiales| Clostridiaceae 1| & Caloramator & & 63.9 & 58.63 & $<0.0001$ & 0.06 \\
\hline & & & Firmicutes| Clostridia| Clostridiales| Catabacteriaceae| & Catabacter\$ & & 67.0 & 68.56 & $<0.0001$ & 0.001 \\
\hline & & & Firmicutes| Clostridia| Clostridiales| Catabacteriaceae| & Catabacter & C. hongkongensis & 64.0 & 68.56 & $<0.0001$ & 0.002 \\
\hline & & & Firmicutes| Clostridia| Clostridiales| Defluviitaleaceae| & Defluviitalea & & 70.2 & 81.32 & $<0.0001$ & 0.07 \\
\hline & & & Firmicutes| Clostridia| Clostridiales| Defluviitaleaceae| & Defluviitalea & D. saccharophila & 71.1 & 81.32 & $<0.0001$ & 0.07 \\
\hline & & & Firmicutes $\mid$ Clostridia| Clostridiales| Peptococcaceae 2| & Pelotomaculum & & 74.5 & 52.36 & $<0.0001$ & 0.07 \\
\hline & & & Firmicutes| Clostridia| Clostridiales| Peptococcaceae 2| & Pelotomaculum & P. propionicicum & 82.6 & 51.30 & $<0.0001$ & 0.01 \\
\hline & & & Firmicutes|Clostridia| Clostridiales|Christensenellaceae| & Christensenella & & 138.5 & 93.14 & $<0.0001$ & 0.06 \\
\hline & & & Firmicutes| Clostridia| Clostridiales| Christensenellaceae| & Christensenella & C. minuta & 170.2 & 93.14 & $<0.0001$ & 0.01 \\
\hline & & & Firmicutes|Clostridia| Clostridiales| Ruminococcaceae| & Saccharofermentans $* \$$ & & 187.6 & 65.13 & $<0.0001$ & 0.005 \\
\hline & & & Firmicutes| Clostridia| Clostridiales| Ruminococcaceae| & Saccharofermentans & S. acetigenes\$ & 189.3 & 65.13 & $<0.0001$ & 0.01 \\
\hline & & & Firmicutes| Clostridia| Clostridiales| Ruminococcaceae| & Sporobacter & & 305.1 & 93.50 & $<0.0001$ & 0.02 \\
\hline & & & Firmicutes| Clostridia| Clostridiales| Ruminococcaceae| & Sporobacter & S. termitidis & 273.1 & 93.50 & $<0.0001$ & 0.07 \\
\hline & & & Firmicutes| Clostridia| Clostridiales| Ruminococcaceae| & Intestinimonas & & 320.8 & 98.70 & $<0.0001$ & 0.003 \\
\hline & & & Firmicutes| Clostridia| Clostridiales| Ruminococcaceae| & Intestinimonas & I. butyriciproducens & 352.4 & 98.70 & $<0.0001$ & 0.002 \\
\hline & & & Firmicutes| Clostridia| Clostridiales| Ruminococcaceae| & Oscillibacter\$ & & 396.2 & 99.76 & $<0.0001$ & 0.03 \\
\hline & & & Firmicutes| Clostridia| Clostridiales| Ruminococcaceae| & Oscillibacter & O. valericigenes & 460.2 & 95.98 & $<0.0001$ & 0.03 \\
\hline & & & Firmicutes| Clostridia| Clostridiales| Eubacteriaceae| & Eubacterium & & 447.6 & 99.88 & $<0.0001$ & 0.06 \\
\hline & & & Firmicutes|Clostridia| Clostridiales| Eubacteriaceae| & Eubacterium & E. coprostanoligenes & 580.5 & 94.44 & $<0.0001$ & 0.01 \\
\hline & $3.111-3.129$ & \multirow{3}{*}{ - Unassigned signal } & $\begin{array}{l}\text { Firmicutes } \mid \text { Clostridia| Clostridiales } \mid \text { Lachnospiraceae| Clostridium } \\
\text { XIVa| }\end{array}$ & Clostridium & C. viride & -133.2 & 90.31 & $<0.0001$ & 0.03 \\
\hline & $3.367-3.437$ & & Firmicutes| Clostridia| Clostridiales| Eubacteriaceae| & Eubacterium & E. rectale* & 266.4 & 98.94 & $<0.0001$ & 0.1 \\
\hline & $3.437-3.496$ & & Firmicutes| Clostridia| Clostridiales| Eubacteriaceae| & Eubacterium & E. rectale* & -470.9 & 98.94 & $<0.0001$ & 0.03 \\
\hline & $3.496-3.587$ & Glucose & Firmicutes|Clostridia| Clostridiales| Eubacteriaceae| & Alkalibaculum & & -20.1 & 74.35 & $<0.0001$ & 0.07 \\
\hline & \multirow{2}{*}{$5.687-5.727$} & \multirow{2}{*}{ Urea } & Firmicutes| Clostridia| Clostridiales| Ruminococcaceae| & Saccharofermentans $* \$$ & & -219.2 & 65.13 & $<0.0001$ & 0.07 \\
\hline & & & Firmicutes| Clostridia| Clostridiales| Ruminococcaceae| & Saccharofermentans & S. acetigenesई & -207.7 & 65.13 & $<0.0001$ & 0.09 \\
\hline
\end{tabular}


Accepted manuscript

\begin{tabular}{|c|c|c|c|c|c|c|c|c|c|}
\hline \multirow{9}{*}{ 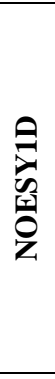 } & \multirow{3}{*}{$3.071-3.111$} & \multirow{3}{*}{ Creatinine } & Firmicutes| Clostridia| Clostridiales| Catabacteriaceae| & Catabacter** & & 79.7 & 68.56 & $<0.0001$ & 0.01 \\
\hline & & & Firmicutes| Clostridia| Clostridiales| Ruminococcaceae| & Saccharofermentans $* \S$ & & 265.0 & 65.13 & $<0.0001$ & 0.03 \\
\hline & & & Firmicutes| Clostridia| Clostridiales| Ruminococcaceae $\mid$ & Oscillibacter\$ & & 567.1 & 99.76 & $<0.0001$ & 0.09 \\
\hline & \multirow{4}{*}{$3.532-3.588$} & \multirow{5}{*}{ Glucose } & Firmicutes|Clostridia| Clostridiales| Ruminococcaceae| & Pseudobacteroides* & \multirow{3}{*}{ P. cellulosolvens } & 28.9 & 19.62 & $<0.0001$ & $<0.0$ \\
\hline & & & Firmicutes $\mid$ Clostridia| Clostridiales| Ruminococcaceae & Pseudobacteroides & & 31.9 & 19.62 & $<0.0001$ & $<0.0001$ \\
\hline & & & Firmicutes| Clostridia| Clostridiales| Clostridiaceae 4| & Salimesophilobacter & & 41.9 & 24.82 & $<0.0001$ & 0.03 \\
\hline & & & Firmicutes|Clostridia| Clostridiales| Clostridiaceae 4| & Salimesophilobacter & \multirow[t]{3}{*}{ S. vulgaris } & 44.5 & 24.82 & $<0.0001$ & 0.03 \\
\hline & $3.588-3.611$ & & Firmicutes| Clostridia| Clostridiales| Ruminococcaceae| & Pseudobacteroides* & & -21.0 & 19.62 & $<0.0001$ & 0.0001 \\
\hline & $9.471-9.511$ & Unassigned signal & Firmicutes $\mid$ Clostridia| Clostridiales| Ruminococcaceae & Clostridium III & & 1038.8 & 98.11 & $<0.0001$ & 0.08 \\
\hline
\end{tabular}

${ }^{1}$ Prevalence of bacterial taxa in the study sample.
${ }^{2}$-value for MaAsLin adjusted for age, sex, BMI, smoking status, and physical activity before Benjamini-Hochberg correction; computed using the Maaslin package on R.
${ }^{3}$ Corrected p-value (Benjamini-Hochberg method, $10 \%$-FDR). Only associations with a Q-value $\leq 0.1$ are presented.

* Taxa found in association with at least 2 NMR signals; $§$ taxa found with both CPMG and NOESY1D 


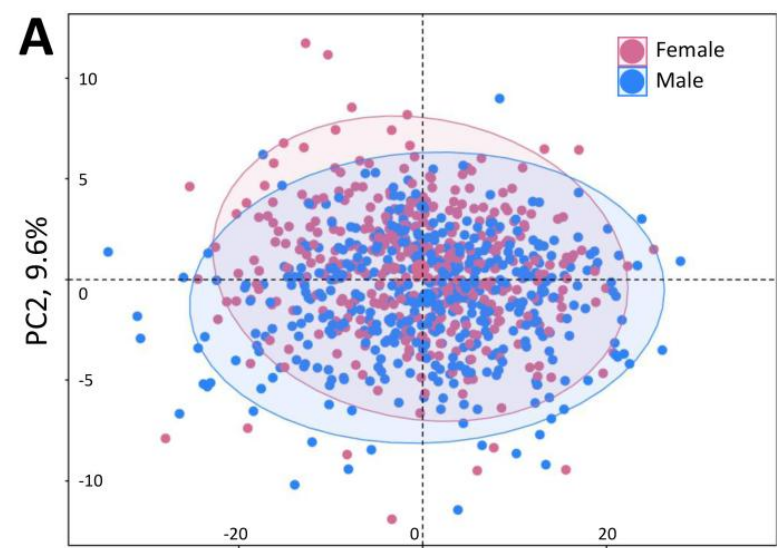

PC1, 52.3\%

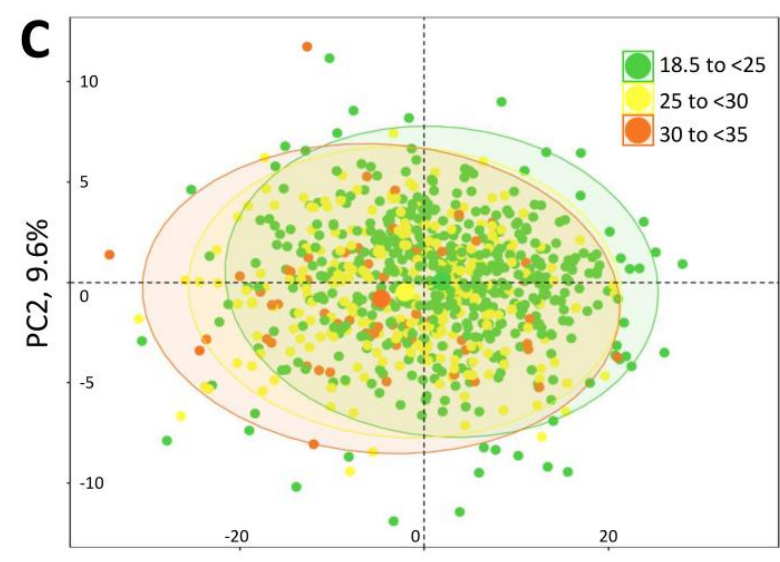

PC1, 52.3\%

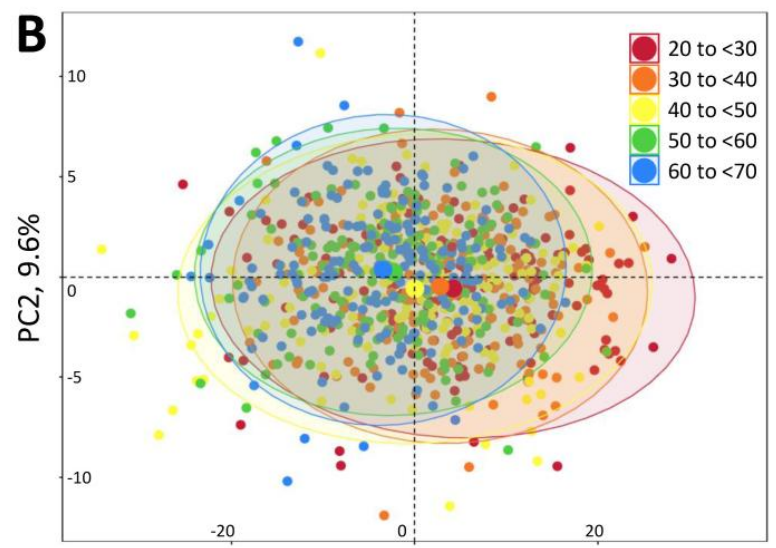

PC1, 52.3\%

Figure 1. Interindividual variation in metabolomic signatures represented by principal components analysis of the CPMG metabolomic dataset, Milieu Intérieur study, France, 2012 (N=846). Each point represents an individual from the study sample. PCA was obtained via the $P C A$ function (package FactoMineR), and plotted and color-coded based on sex (A), age (B), and BMI (C) via the fviz_pca_ind function (package FactoExtra). Concentration ellipses (95\%) are shown. Percentages on the axes represent the proportion of variation explained by the two first components of the PCA. 


\begin{tabular}{|c|c|c|c|c|}
\hline \multirow[b]{2}{*}{ Sequence } & \multirow[b]{2}{*}{ Shift (ppm) } & & \multicolumn{2}{|c|}{ Richness } \\
\hline & & & Observed & Chao1 index \\
\hline \multirow{24}{*}{$\sum_{0}^{O}$} & $0.567-0.607$ & Unassigned signal & * & - \\
\hline & $1.207-1.311$ & Lipoproteins, $-\mathrm{CH}_{2}$ & $\star \star$ & * \\
\hline & $1.521-1.527$ & $\mathrm{CH}_{2} \mathrm{CH}_{2} \mathrm{COOC}$ & $\star \star$ & * \\
\hline & \multirow{2}{*}{$\begin{array}{l}2.151-2.166 \\
2.166-2.167 \\
\end{array}$} & \multirow{2}{*}{ Lipid, $-\mathrm{CH}_{2}=\mathrm{C}=\mathrm{C}-$} & * & * \\
\hline & & & $\star \star$ & * \\
\hline & $2.167-2.264$ & Acetone, $\mathrm{CH}_{2} \mathrm{CO}$ & ** & * \\
\hline & $2.551-2.571$ & Methylamine & ** & * \\
\hline & $2.831-2.848$ & Citrate $/=\mathrm{CH}-\mathrm{CH}_{2}-\mathrm{CH}=$ & * & - \\
\hline & $3.071-3.111$ & Creatinine & $\star \star$ & * \\
\hline & $3.167-3.269$ & Choline and glucose & * & - \\
\hline & $3.311-3.312$ & Glucose & * & - \\
\hline & $3.616-3.711$ & Glutamine & * & * \\
\hline & $5.127-5.167$ & \multirow{4}{*}{ Unassigned signal } & ** & * \\
\hline & \multirow{2}{*}{$\begin{array}{l}5.864-5.889 \\
5.889-5.969\end{array}$} & & * & - \\
\hline & & & $\star \star$ & - \\
\hline & $6.492-6.527$ & & ** & * \\
\hline & $6.728-6.767$ & Proteins & ** & * \\
\hline & $6.857-6.943$ & Tyrosine & * & * \\
\hline & 6.912-6.943 & \multirow{2}{*}{ Proteins } & ** & * \\
\hline & 6.943-6.968 & & ** & * \\
\hline & $7.508-7.539$ & \multirow{2}{*}{ 3-methylhistidine } & $\star \star$ & - \\
\hline & $7.629-7.647$ & & $\star \star$ & * \\
\hline & $7.647-7.687$ & 1-methylhistidine & ** & * \\
\hline & $7.687-7.772$ & Histidine & ** & * \\
\hline \multirow{8}{*}{ 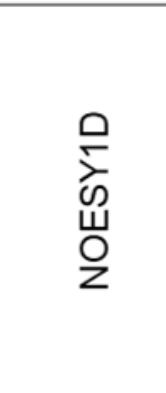 } & 4.191-4.214 & \multirow{6}{*}{ Unassigned signal } & $\star \star$ & $\star \star$ \\
\hline & $5.830-5.835$ & & ** & * \\
\hline & $\overline{5.861-5.887}$ & & ** & ** \\
\hline & \multirow{3}{*}{$\begin{array}{l}5.887-5.965 \\
5.965-5.966 \\
6.486-6.526\end{array}$} & & $\star \star$ & $\star \star$ \\
\hline & & & $\star \star$ & $\star \star$ \\
\hline & & & * & - \\
\hline & $6.726-6.766$ & Proteins & $\star \star *$ & $\star \star$ \\
\hline & $7.646-7.686$ & 1-methylhistidine & * & - \\
\hline
\end{tabular}

Spearman $\rho$

$-0.13$

Figure 2. Associations between NMR variables and $\alpha$-diversity indexes (observed richness and Chao1 estimate of richness) from Spearman partial correlations adjusted for age, sex, BMI, smoking status, physical activity, and sequencing depth, Milieu Intérieur study, France, 2012 (N=846). Qvalues were obtained applying a multiple-testing correction (Benjamini-Hochberg false discovery rate method). Only associations with $\mathrm{Q}$-value $\leq 0.1$ for observed richness, as well as subsequent associations with Chao1 richness are presented. '** ' $\mathrm{P}$-value $\leq 0.003$ and $\mathrm{Q}$-value $\leq 0.05$; ' * ' $\mathrm{P}$ value $\leq 0.01$ and $\mathrm{Q}$-value $\leq 0.1$; ' ${ }^{-}$'P-value $\leq 0.05$ and $\mathrm{Q}$-value $>0.1$. Corresponding values are shown in Supplementary Table 7. 